\title{
ENDOMETRIOSIS EN EL CANAL INGUINAL: REPORTE DE CASO
}

\section{Quirós Alpízar, José Luis y Corrales González, Daniela}

Departamento de Anatomía de la Escuela de Medicina de la Universidad de Costa Rica, San Pedro, San José, Costa Rica.

\begin{abstract}
Resumen: Endometriosis se define como la presencia de tejido glandular y estromal endometrial fuera de la cavidad uterina y este tejido ectópico es dependiente de la estimulación estrogénica. Usualmente se presenta en ovarios, fondos de saco, ligamento ancho, ligamento uterosacro, útero, trompas de Falopio, tracto gastrointestinal y geniturinario. Sin embargo, puede presentarse en otras localizaciones, como en el canal inguinal. Prevalece en $10 \%$ de las mujeres en edad reproductiva. Existen muchos factores contribuyentes a esta patología como la herencia, edad, anomalías anatómicas del sistema reproductor femenino, paridad, alteraciones del ciclo menstrual, uso de anticonceptivos orales, factores ambientales. Fisiopatológicamente hay muchas teorías descritas entre estas la menstruación retrógrada que podría explicar la etiología del caso que se describe en este artículo. El síntoma más frecuente es el dolor pélvico y se ha asociado también la infertilidad. El diagnóstico de elección se realiza mediante laparoscopía o laparotomía, acompañado de confirmación histológica de macrófagos con hemosiderina, epitelio endometrial y glándulas o estroma endometrial. Se ha observado transformación maligna en 0.6-0.8\% de las mujeres que presentaron endometriosis ovárica. Dentro del tratamiento médico los análogos de la hormona GNRH son ampliamente usados, mientras que se utiliza menos el danazol por sus efectos androgénicos, el uso combinado de AINES y anticonceptivos orales se aplica cuando las pacientes asocian mucho dolor pélvico. El tratamiento quirúrgico consiste en la ablación del tejido endometrial ectópico a través de laparoscopia.
\end{abstract}

Palabras clave: endometriosis, canal inguinal, inmunohistoquímica, receptores de estrógeno, CD10 


\title{
ENDOMETRIOSIS IN THE INGUINAL CHANNEL: A CASE REPORT
}

\begin{abstract}
Endometriosis is defined as the presence of functioning endometrial glands and stromal tissue outside of uterine cavity, which is estrogen dependent. It's usually seen in ovary, uterosacral ligament, uterus, fallopian tubes, gastrointestinal tract and genitourinary system. However we describe a case in the inguinal channel. It prevails in $10 \%$ of women in reproductive age. There are a lot of factors that contribute to this pathology like inheritance, age, female's reproductive system's anatomic anomalies, parity, alterations of the menstrual cycle, use of oral contraceptives and ambient factors. There are a lot of physiopathological theories; one of them is retrograde menstruation that could explain the etiology of the case presented. The most frequent symptom is pelvic pain and infertility has also been associated. The best way of diagnosis is laparoscopy or laparotomy, along a histological confirmation of macrophages with hemosiderin, endometrial epithelium and endometrial glands or stroma. Malignant transformation has been observed in 0,6-0,8\% of women that presented ovaric endometriosis. GnRH analogues are broadly used as medical treatment, while danazol is less used because of its androgenic effects. NSAIDs combined with oral contraceptives is used to reduce pelvic pain. Chirurgical treatment consists on endometrial ectopic tissue ablation trough laparoscopy.
\end{abstract}

Key words: endometriosis, inguinal channel, immunohistochemistry, estrogen receptors, CD10

\section{INTRODUCCIÓN}

Este artículo presenta un caso clínico poco descrito de endometriosis.

La endometriosis es una enfermedad ginecológica frecuente en mujeres en edad reproductiva, comúnmente a nivel de ovarios, sin embargo, la presentación puede ser muy diversa. Se describen varios factores de riesgo como la edad, las características reproductivas, los hábitos personales y los factores hereditarios directamente relacionados con el desarrollo de la enfermedad [1]. La endometriosis es la causa más común de dolor pélvico crónico. La prevalencia estimada es de $10 \%$ mujeres [2] y $25 \%$ de las mujeres con infertilidad [3].

\section{CASO CLÍNICO}

J. B. S., paciente femenina de 48 años, con histecterectomía abdominal y salpingo-oforectomía izquierda en 2004 por leiomiomatosis del cuerpo uterino con sangrado uterino anormal, con ultra sonido
(US) pélvico en 2009 sin hallazgos patológicos. Además cuadrantectectomía mamaria derecha por hiperplasia ductal con atipia en 2010, en tratamiento con tamoxifeno, en 2012 se realiza biopsia excisional por nódulo en mama derecha con microcalcificaciones. Antecedente ginecoobstétricos: G2,P2,C1,A0, no utiliza anticonceptivos orales.

En noviembre 2011 la paciente se realiza un ultrasonido abdominal de rutina que encontró un área hipoecoica con centro líquido de $52 \times 15 \mathrm{~mm}$, como hallazgo incidental, en región inguinal derecha junto a paquete vasculonervioso, compatible con adenopatía con centro necrótico versus hematoma residual antiguo, con leve efecto de masa. La paciente en abril del 2012 presenta un nódulo inguinal derecho palpable de $47 \times 17 \times 21 \mathrm{~mm}$, no doloroso a la palpación, niega sintomatología localizada sin embargo 5 años de evolución de dolor pélvico y mastalgia. Se realizan marcadores tumorales los cuales se encuentran normales. 
Se realiza cirugía excisional del canal inguinal derecho y se diseca el nódulo junto al ligamento redondo.

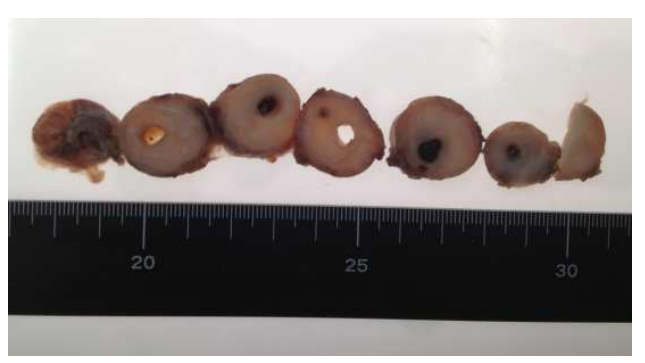

Figura 1. Cortes seriados de Nódulo fibrótico con sangrado central

Se realiza biopsia que reporta macroscópicamente formación nodular de consistencia elástica de $40 \times 20 \mathrm{~mm}$, al corte la lesión es blanquecina de aspecto arremolinado con centro gelatinoso café oscuro, como se observa en la figura 1.

Microscópicamente, se identificó zona de fibrosis en la periferia del nódulo, en la región central se identifica, cavidad con restos hemáticos, adyacente a la cavidad hay glándulas revertidas por epitelio cilíndrico simple rodeadas por un estroma celular; lo anterior descrito se observa la figura 2 en $a$ y $b$. En $c$ se observan células glandulares que muestran núcleos positivos para el anticuerpo de receptores de estrógenos. En $d$ el estroma presenta tinción inmunohistoquímica positiva para CD10. Se diagnóstica como nódulo fibrótico con endometriosis central.

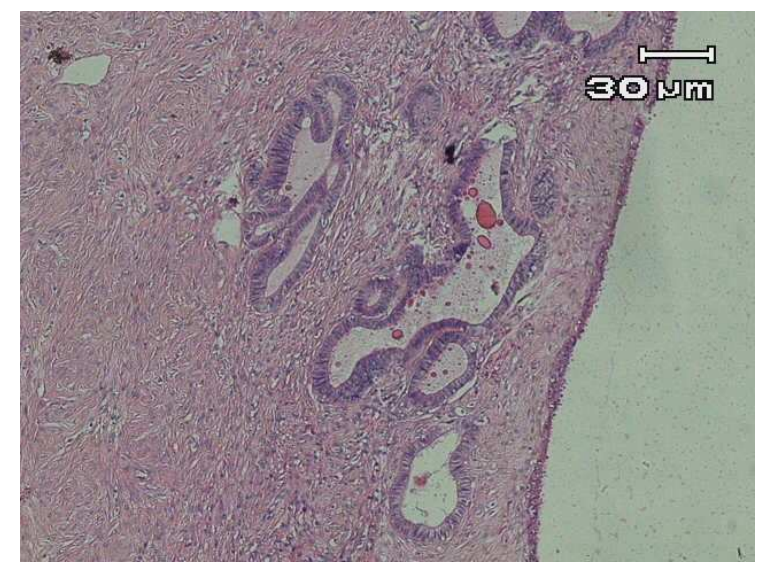

$a$

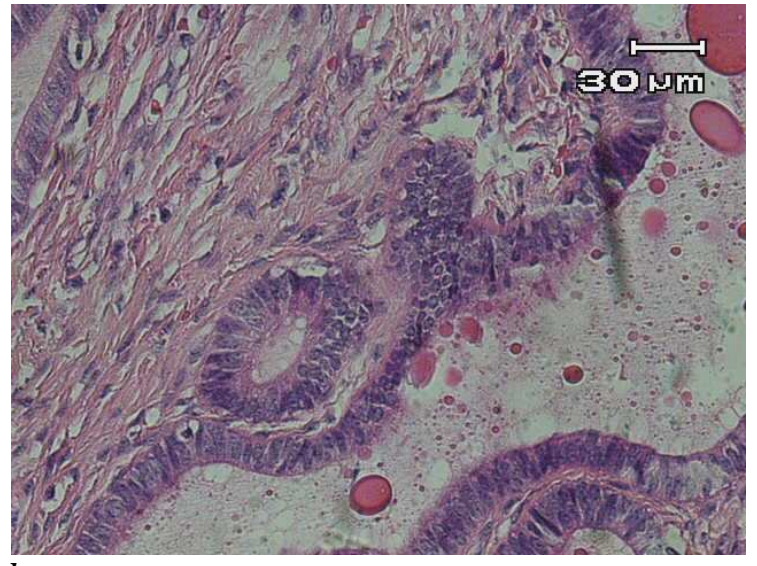

$b$

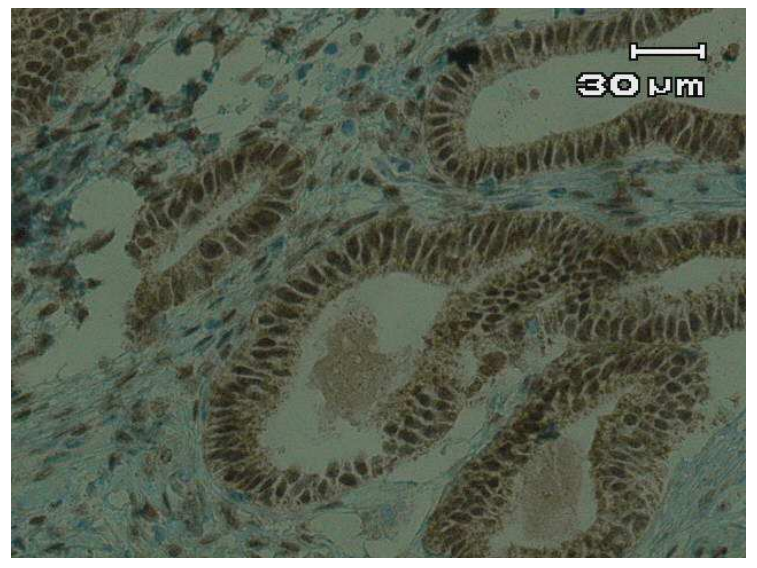

C

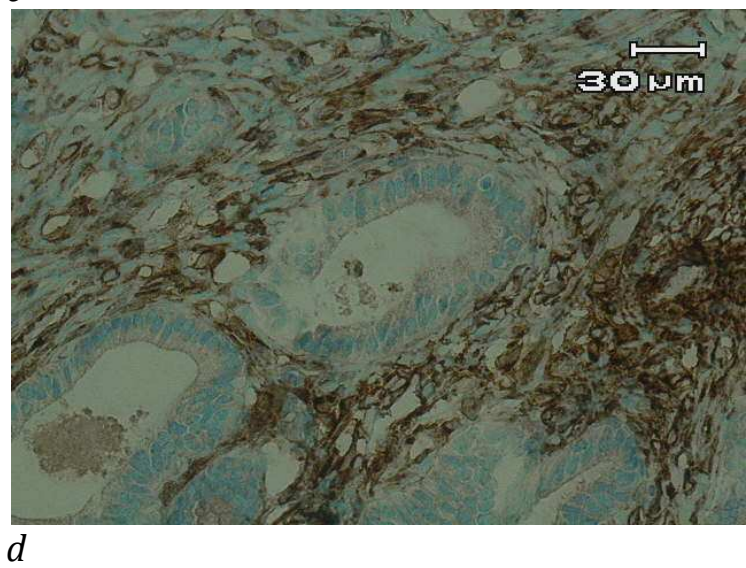

Figura 2. Microfotografías de cortes histológicos del nódulo fibrótico.

\section{DEFINICIÓN Y EPIDEMIOLOGÍA}

La endometriosis se define como la presencia de tejido glandular y estromal endometrial fuera de la cavidad uterina, siendo este tejido ectópico dependiente de la estimulación estrogénica.

Revista electrónica publicada por el Departamento de Farmacología de la Escuela de Medicina de la Universidad de Costa Rica, 2060 San José, Costa Rica. ${ }^{\circledR}$ All rights reserved. Licensed under a Creative Commons Unported License. 
Esta enfermedad afecta entre 5 y $10 \%$ de mujeres en edad reproductiva. Se diagnóstica principalmente en mujeres que sufren de dolor crónico o infertilidad, por medio de laparoscopia. Sin embargo es posible que se encuentre subdiagnosticada [4]. Es importante aclarar que se ha presentado casos de endometriosis en hombre como lo reporta Simsek et al en un caso de endometriosis inguinal [5]. Existen muchos factores contribuyentes a esta patología como la herencia, edad, anomalías anatómicas del sistema reproductor femenino, paridad, alteraciones del ciclo menstrual, uso de anticonceptivos orales, factores ambientales. La edad es el único factor que se relaciona de manera consistente con esta enfermedad alcanzando el máximo riesgo relativo en mujeres de 40-44 años [1]. Diversos estudios indican que la probabilidad de endometriosis es de 3 a 10 veces mayor cuando existen antecedentes familiares de primer grado; además, en mujeres con obstrucción a nivel del tracto vaginal existe un riesgo aumentado de endometriosis. Otros factores asociados son: la multiparidad o el extremo opuesto la nuliparidad o bien periodos intergénicos largos, así como ciclos menstruales irregulares con fase menstrual muy prolongada [4]. Chapron et al, realizó un estudio transversal de 566 pacientes con diagnóstico quirúrgico de endometriosis ovárica, peritoneal e infiltrante profunda; para evaluar si estaba asociado al uso de anticonceptivos orales. Concluyeron que las pacientes que usaban anticonceptivos orales por dismenorrea, asociaban principalmente endometriosis profunda infiltrativa, cabe destacar que aclaran que no significa que los anticonceptivos orales aumenten el riesgo de endometriosis [6]. Por otro lado, el uso cíclico de anticonceptivos orales monofásicos puede prevenir o ayudar en el manejo de la endometriosis. Esto está basado en que el flujo menstrual es menos denso en pacientes que usan anticonceptivos lo que probablemente permite una disminución en la menstruación retrógrada. Más recientemente, se ha encontrado que los anticonceptivos orales trifásicos suprimen la endometriosis pero la enfermedad reaparece después de suspender esta medicación. En cuanto a factores ambientales no hay asociación con tabaco, sí se ha asociado a uso de dietiletilbestrol intrauterino y en países industrializados a componente contaminante dioxina [1]. En el caso clínico la paciente solamente tiene la edad y el antecedente de ciclos menstruales prolongados por la miomatosis uterina previa a la histerectomía, ninguno de los otros factores de riesgo se documentaron.

Es importante recordar que en orden de frecuencia la endometriosis se encuentra en ovarios, fondos de saco, ligamento ancho, ligamento uterosacro, útero, trompas de Falopio, tracto gastrointestinal y geniturinario [9]. Tejido endometrial ectópico de pared abdominal ocurre en $0.03 \%$ a $1 \%$ de las mujeres con previa cirugía obstétrica o ginecológica [3]. Dentro de las cirugías que se han asociado con hallazgo de endometriosis están histerectomías, ligadura de trompas uterinas, cesáreas, episiotomías y laparoscopía [16].

El dolor pélvico es el síntoma más frecuente principalmente durante el periodo premenstrual y al término de la menstruación. Además, puede aparecer dolor de espalda, dispareunia, dolor a la defecación, dolor al cambio de posición y disuria de aparición cíclica. La asociación de endometriosis con infertilidad se debe a las adherencias en la cavidad peritoneal, lo cual distorsiona la anatomía pélvica y altera la liberación adecuada del óvulo; sin embargo, los trastornos anatómicos no son el único motivo de la infertilidad, ya que se ha descrito que la endometriosis causa baja reserva ovárica y de la calidad de óvulo (10). Lesiones que involucran el tracto urinario o intestino resultan en hematuria o hematoquecia en el periodo premenstrual. Implantaciones cerca de superficies externas de cérvix, vagina, recto, uretra puede causar dolor o sangrado rectal o por uretra. Adhesiones de endometriosis podrían causar leve malestar [7]. En el caso analizado anteriormente la endometriosis inguinal dentro del nódulo fibrótico no producía síntomas como dolor o sangrado, presentaba efecto de masa, por lo tanto no se puede descartar que esta paciente presentaba focos endometriósicos intrapélvicos.

\section{FISIOPATOLOGÍA/ETIOPATOGENIA}

Existen muchas complejas teorías sobre el origen de endometriosis, aun no hay consenso. En la actualidad existen cuatro teorías para explicar la patogénesis de la endometriosis: metaplasia celómica, menstruación retrógrada con trasporte de células endometriales, diseminación linfática y vascular, y teoría de restos embrionarios. Incluso una combinación de estas teorías [7]. Por otro lado recientemente se habla de un fallo de mecanismos inmunes a la destrucción de tejido ectópico o una diferenciación anormal del tejido endometrial, han sido sugeridos como mecanismos subyacentes asociados al defecto del tejido estromal con aumento de estrógenos y prostaglandinas resistente al estímulo de progestágeno [4]. A continuación un pequeño resumen de estas teorías: 
- Menstruación retrógrada: fragmentos viables de endometrio pasan a través de trompas uterina a la cavidad pélvica con implantaciones peritoneales, ovarios, fondo de saco, vejiga, episiotomía e incisiones, esta teoría se ha observado en mujeres con estenosis cervical y septo vaginal transverso, también en mujeres con prolongado flujo menstrual. Sin embargo esta teoría no explica la endometriosis pulmonar, cerebral, tejidos blandos o en hombres [4]. La paciente del caso clínico presentaba previo al 2004 flujo menstrual abundante y obstrucción a nivel de cuerpo uterino por la leiomiomatosis.

Metaplasia celómica: fue considerada la primera teoría que explicaba la patogenia de la endometriosis, se sugirió que la endometriosis se originaba en la membrana celómica a través de un proceso de metaplasia seguido de una inducción metaplásica. El epitelio celómico, que surge de una capa decélulas epiteliales del conducto mulleriano, también se diferencia en epitelio pleural y peritoneal y en células de la superficie de los ovarios. Esta teoría explica la endometriosis que se presenta en hombres, en mujeres en etapa prepuberal, en aquellas que jamás han menstruado y en adolescentes sin anormalidades del conducto de mullerianos [1].

- Diseminación linfática y vascular: se ha postulado la viabilidad de las células endometriales que entraban a través de la membrana basal de los vasos sanguíneos y linfáticos. Estas células endometriales mediante la formación de microémbolos provocarían el desarrollo de la enfermedad en lugares a distancia [1].

- Restos embrionarios: la identificación de restos del conducto paramesonéfrico en el ligamento ancho y porciones antero-laterales de la vagina y el cérvix, que podrían dar lugar a la endometriosis. La presencia de estos restos embrionarios puede explicar la existencia de endometrio ectópico. Áreas adyacentes a los conductos mullerianos y duplicaciones del sistema mulleriano, permitirían que células de origen mulleriano se conviertan en endometrio funcionante. Sin embargo, la presencia de los mismos no se ha encontrado en la pelvis o en la cavidad torácica. Si consideramos esta hipótesis, la endometriosis debería aparecer inmediatamente después de la menarquia, cuando la estimulación hormonal se ha iniciado [1].

Inmunidad humoral y celular: juega un papel importante en la patogénesis de la endometriosis. La reducción de células NK y deficiente inmunidad celular no es capaz de reconocer el tejido endometrial ectópico y por lo tanto permite que se implante y crezca en otro lugar, gracias a factores de crecimiento y citoquinas secretadas por macrófagos. Además, se ha investigado que el alelo HLA-B7 inhibe la actividad citotóxica de células NK, esto se ha visto en $7 \%$ de antecedentes familiares de primer grado. (7) Las citoquinas y factores de crecimiento asociadas a la endometriosis son VEGF, PGE2, TNF- $\alpha$ e IL-1, las cuales promueven la producción de IL-8 y RANTES. Además, en la fisiopatología de la endometriosis, los macrófagos hallados en la cavidad peritoneal normal sin endometriosis secretan metaloproteinasas (MMPs) y receptores barrendero (scavenger receptor) como lo son receptores barrendero CD36, para degradar tejidos ectópicos, pero en endometriosis la capacidad de degradación de estos está disminuida [8].

\section{DIAGNÓSTICO}

El diagnóstico de elección de la endometriosis se realiza mediante laparoscopía o laparotomía, acompañado de confirmación histológica de macrófagos con hemosiderina, epitelio endometrial y glándulas o estroma endometrial.

El antígeno carcinoembrionario-125 en suero (CA-125), que se ha visto elevado en endometriosis; y la imagen por resonancia magnética han demostrado escaso valor diagnóstico, pero son losmétodos con los que se puede realizar el diagnóstico no invasivo. El CA-125, a pesar de inespecífico, se puede utilizar para monitoreo o seguimiento de recurrencias, en el presente caso clínico este marcador estaba normal [7]. También otros marcadores séricos como el antígeno carcinoembrionario-19-9 en suero (CA 19-9), IL6, TNF alfa en el líquido peritoneal se han usado, aunque desafortunadamente esta última prueba es un procedimiento invasivo que requiere obtener el líquido peritoneal. El ultrasonido intravaginal ha sido el de mayor uso en el diagnóstico de lesiones retroperitoneales y uterosacras, pero no tiene la exactitud para identificar lesiones peritoneales o endometriomas pequeños [10].

Además, se ha estado estudiando las bases genéticas de la endometriosis, Govatati et al, secuenciaron el genoma mitocondrial de tejido endometrial ectópico y eutópico y concluyen que la exploración del genoma mitocondrial es un marcador diagnóstico de endometriosis, ya que hallaron en el ADN mitocondrial de pacientes con endometriosis deleciones e inestabilidad microsatélite mitocondrial del tejido ectópico por un aumento de especies reactivas de oxígeno (ROS) y además se halló un halogrupo M5 con riesgo aumentado de incidencia de endometriosis [11]. Esto es un comienzo de detección con genética molecular lo cual aún no está en la práctica accesible 
para el diagnóstico. Sin embargo a pesar de tantos estudios génicos no se ha encontrado con un gen que se relacione claramente con endometriosis [12].

\section{TRANSFORMACIÓN ENDOMETRIOSIS \\ MALIGNA DE}

Según datos reportados de casos aproximadamente el $1 \%$ de las mujeres con endometriosis tiene lesiones bajo trasformación maligna, de estos $80 \%$ a nivel ovárico y $20 \%$ extragonadal. L a transformación maligna se ha observado como una complicación de endometriosis en $0.6-0.8 \%$ de las mujeres que presentaron endometriosis ovárica. Estos procesos malignos se han clasificado en 3 grupos:

- Cáncer epitelial de ovario como el adenocarcinoma endometroide y el de células claras.

- Tumores de tipo mulleriano como tumor borderline de tipo mulleriano $y$ tumor borderline seroso.

- Sarcomas como adenosarcoma, sarcoma estromal endometrial

Se han asociado múltiples alteraciones genéticas a los casos de transformación maligna post endometriosis por estímulo de estrés oxidativo, como mutaciones en p53, activación de KRAS, inactivación de ARID1A, activación PIK3CA y HNF-1 $\beta$. Otras mutaciones reportadas son KRAS, BRAF, ERBB2, MMBT y ESS [13].

\section{TRATAMIENTO}

\section{Tratamiento quirúrgico}

El tratamiento quirúrgico se hace mediante laparoscopía y se efectúa al mismo tiempo que el diagnóstico; puede ser conservador, cuando se preservan ovarios y útero, o radical, cuando se extirpan. Se prefiere siempre tratamiento conservador ya que la endometriosis es de progresión lenta, rara vez se maligniza y al tratarse permite el embarazo, que también ejerce un efecto benéfico sobre ella. Además desaparece con la menopausia y las probabilidades de recidiva con terapia hormonal sustitutiva son bajas. El tratamiento quirúrgico es útil en etapas iniciales y avanzadas de la enfermedad. (14)

Indicaciones de cirugía:

1. Pacientes con dolor pélvico:

a. No responde a tratamiento médico o tiene contraindicaciones.

b. Con evento agudo anexial c. Con invasión severa a intestino, vejiga, uréteres, nervios pélvicos

2. Pacientes que tienen o con sospecha de endometrioma que asocian dolor crónico o efecto de masa.

\section{TRATAMIENTO MÉDICO}

Hormonal: dentro de estos se utilizan los siguientes:

Estrógenos y progestágenos: etinilestradiol en dosis de 20 a $35 \mu \mathrm{g}$ y progestágenos diversos en es quemas continuos de una tableta diaria.

Progestágenos de depósito: acetato de medroxiprogesterona intramuscular en dosis de 100 mg cada dos semanas por cuatro dosis, seguidos de 200 mg mensuales por cuatro dosis más.

Progestágenos orales: 30 mgdiarios por 3 meses o linestrenol, $10 \mathrm{mg}$ diarios por 6 meses.

Progestágenos intrauterinos: levonorgestrel, lo que tiene su mayoraplicación en casos de involucramiento rectovaginal.

Danazol:800 mg diarios, divididos en cuatro dosis al día por seis meses. Este tiene efectos androgénicos.

Agonistas de GnRH: Los esquemas más recomendables son a base del acetato de leuprolida de depósito en dosis de $3.75 \mathrm{mg}$ cada 4 semanas por seis meses o el acetato de goserelina en una píldora biodegradable para administración subcutánea mensual, así como también el acetato de nafarelina en dosis de $200 \mu \mathrm{g}$ diarios por vía intranasal.

Antagonistas de GnRH: se recomienda la administración secuencial de la dosis única semanal durante 8 semanas, se reporta regresión en alrededor de $60 \%$ de los casos, sin síntomas de hipoestrogenismo severo.

Antiprogestágenos: $50 \mathrm{mg}$ diarios por lapsos de 3 a 6 meses, con lo que se logra inhibirla ovulación y romper la integridad endometrial.

Inhibidores de aromatasas: primera opción en el tratamiento de endometriois en la posmenopausia, ya que aquí la fuente de producción de estrógenos es extragonadal [14].

Alterativa a tratamiento hormonal: para pacientes con contraindicaciones se han utilizado: pentoxifilina, leflunomida, etanercept, infliximab, levimasol y loxoribina, proteína fijadora de TNF, interferón, inhibidores de la ciclooxigenasa y otros medicamentos en etapa de investigación, entre los que destacan los inhibidores de matriz metaloproteínasas y diferentes agentes antiangiogénicos. El 5-fluorouracilo ha 
demostrado disminución en la proliferación de células endometriales, in vitro, en paciente con endometriosis profunda y endometriomas [14].

Las opciones de tratamiento deben discutirse con la pareja, quienes deben comprender que sin cirugía radical ablativa las recidivas son frecuentes y que los tratamientos conservadores o quirúrgicos tienen indicaciones específicas según el estadio en que se encuentre la enfermedad. La terapéutica se planea de acuerdo con los deseos de la paciente de lograr un embarazo, para tratar dolor, tumoraciones pélvicas, sangrados genitales anormales y dispareunia [14].

\section{PRONÓSTICO}

La endometriosis puede estabilizarse o tener regresión por sí sola, ya que en $25 \%$ de las pacientespuede haber resolución de la enfermedad. Por otro lado, se ha observado que la endometriosis puede recurrir incluso después del tratamiento. Además, las mujeres posmenopáusicas con antecedentes de endometriosis pueden presentar nuevamente este padecimiento durante el uso de la terapia hormonal, por lo tanto, en ambos casos se debe tener cuidado en la administración de estrógenos cuando estén indicados [10].En cuanto a recidiva hay ciertos factores implicados en la recurrencia tras la extirpación laparoscópica de endometriomas ováricos, dentro de estos factores están la dismenorrea y dispareunia de alto grado, previas a la cirugía según un estudio realizado por López et al. que concluyó que tanto recidiva sintomática como ecográfica, se presentó en pacientes con estos factores previo a la cirugía [15]. No se describen casos de recidiva de endometriosis de la pared abdominal como en el caso de nuestra paciente al inicio descrito.

\section{CONCLUSIONES}

La endometriosis es una patología que a pesar de ser más frecuente a nivel ovárico, tiene presentaciones extragonadales como por ejemplo en el canal inguinal. Dentro de los factores condicionantes de endometriosis del presente caso tenemos primero, el antecedente de cirugía previa; y segundo, leiomiomas uterinos que favorecen la menstruación retrógrada conllevando a la diseminación de focos de endometriosis a la cavidad abdominal y de ahí al canal inguinal.
Se concluye que no toda endometriosis es dolorosa como en este caso y dentro de los principales diagnósticos diferenciales que hay que tener en cuenta están adenopatías palpables a nivel inguinal y hernia inguinal.

\section{REFERENCIAS}

1. García M. et al. Endometriosis. Departamento de Ginecología y Obstetricia. Clínica Universidad de Navarra. Rev Med Univ Navarra. 2009; 53 (2):47.

2. Gerlinger, et al. Treatment of endometriosis in different ethnic populations: a meta-analysis of two clinical trials. Biomedical central Women's Health 2012;12 (9): 10-23.

3. Simoglu et al. Abdominal wall endometrioma mimicking an incarcerated hernia. International Journal of General Medicine 2012; 5: 569-571.

4. Leyland, N. Endometriosis: Diagnosis and Management. Journal of Obstetrics and Gynaecology Canada. 2010 July; 32 (7):1-27.

5. Simsek et al. An Unusual Cause of Inguinal Hernia in a Male Patient: Endometriosis. Gut and Liver, Vol. 6, No. 2, April 2012, pp. 284-285.

6. Chapron, et al. Oral contraceptives and endometriosis: the past use of oral contraceptives for treating severe primary dysmenorrhea is associated with endometriosis, especially deep infiltrating endometriosis. Human Reproduction. 2011;26(8):2028-2035.

7. De Cherney, A. et al. Chapter 43. Endometriosis Current Diagnosis \& Treatment Obstetrics \& Gynecology, Tenth Edition, 2007.

8. Herington et al. Immune interactions in endometriosis Expert Rev Clin Immunol. Author manuscript; available in PMC 2012 July 1.

9. Kondo W, Ribeiro R, Trippia C, Zomer MT Endometriose profunda infiltrativa: distribuição anatômica e tratamento cirúrgico. Rev Bras Ginecol Obstet. 2012; 34(6):278-84. 
10. Hernández, M. Endometriosis. Base fisiopatogénica para el tratamiento Rev Med Inst Mex Seguro Soc 2009; 47 (1): 57-60.

11. Govatati et al Mitochondrial Mutations in Endometriosis A Study in South Indian Population. July 2012; 7(7):1-12.

12. Rahmioglu, N. Insights into Assessing the Genetics of Endometriosis Curr Obstet Gynecol Rep. 2012; 1:124-137.

13. Higashiura, v. Et al, Malignant transformation of endometriosis. Oncology letters. 2012; 4: 3-9.

14. Ginecología y Obstetricia de México Diagnóstico y tratamiento de endometriosis Ginecol Obstet Mex 2011;79(11):697-710.

15. Lopez et al. Recidivas de endometriomas ovaricas tras cirugia laparoscopia. Rev Chil Obstet Ginecol 2011; 76(6): 380-388.

16. Singh, A. Umbilical endometriosis mimicking as papilloma to general surgeons: A case report. Australasian Medical Journal 2012; 5(5):272274.

\section{INFORMACION DE AUTOR:}

Quirós Alpizar, José Luis

E-mail:ㅁ.quiros@gmail.com

Médico patólogo, Departamento de Anatomía, Universidad de Costa Rica.

Hospital Maximiliano Peralta de Cartago.

Caja Costarricense de Seguro Social.

Corrales González, Daniela

E-mail: danielacorrales2004@yahoo.com

Médico General, Departamento de Anatomía, Universidad de Costa Rica. 